\title{
The Space Telescope Imaging Spectrograph
}

\author{
Bruce E Woodgate
}

NASA/Goddard Space Flight Center

\section{Instrument}

The Space Telescope Imaging Spectrograph (STIS) is a second generation instrument to be installed into the Hubble Space Telescope (HST) in-orbit 5-9 years after the first launch. Together with the infra-red instrument, it will provide a large increase in capability of the observatory, and be able to replace a first generation that had failed or degraded.

The STIS will be able to replace both first generation instruments with an increase in angular and/or spectral coverage of a factor 200-2000, and will operate in the spectral range 1050-11000A. Also it will provide broad-band camera capability over this range, and high-speed photometry.

The capability is centered around two kinds of $2048 \times 2048$ pixel detectors, Multi-Anode Microchannel Arrays (MAMAs) in the UV and charge-coupled devices (CCDs) in the visible and near-IR. Interchangeable spectral formats allow selection of spectral resolving powers between 80 and 140000 and a field-of-view up to 50 arc-sec. The MAMA detectors allow UV spectrographic and camera observations without obscuration due to scattering from the visible. The operating modes (spectral range, resolving power, effective area and angular range) are shown in the Table, and the optical arrangement to accomplish them in the Figure. The mode is selected by rotating the grating wheel which inserts the appropriate optic (grating, prism or mirror) and steers the beam to the next optic (grating, mirror or detector). The slit wheel is also rotated to match the mode. In this way the detector (spectral band) and resolving power as shown in the Table are selected. For each mode, the full angular resolution of the HST of 0.05 arc sec is maintained with 2-pixel sampling. Limited spectral selectivity in the camera modes will be provided in each of the 4 spectral bands by inserting 4 filters each covering a quadrant of the $50 \mathrm{x}$ 


\section{Scientific Objectives}

The capabilities of STIS will allow astronomers from a broad range of disciplines to pursue programs that are not feasible or are very sharply limited in scope with the first generation of HST instruments. These investigations will be aimed at a deeper physical understanding of the origin and evolution of galaxies and their nuclear activity, of the nature and energy balance of the material comprising the interstellar and intergalactic medium, of the dynamical processes of star formation and subsequent variable stellar phenomena, and of the origin and evolution of the solar system. These topics may be illustrated by examples of observations which could answer questiong of great current interest, while recognizing that the HST first generation cameras and spectrographs will have discovered entirely new phenomena and questions for us to investigate.

Absorption lines in the spectra of quasars enable us to study the evolution of the intergalactic medium, intervening clusters of galaxies, and haloes and disks of galaxies as a function of cosmic time. The strong absorption lines fall in the UV at the absorbing object, and can be studied from the ground only at high redshift, where very few of the objects can be detected in emission. An evolutionary comparison with more nearby objects can only be made by systematic studies in the UV. By combining simultaneous broad spectral coverage and fairly high spectral resolving power (20000), STIS will allow sensitive searches for absorption features over a wide redshift range. For example, Lyman alpha systems may be searched for over the redshift range $0<z<1.5$ in two exposures. A resolving power above 10000 is required to find low density clouds in Lyman alpha, to find weak lines of low abundance species in the denser clouds, to separate weak components such as deuterium from hydrogen, and to separate velocity components. For particularly narrow features in galactic disks a resolving power of 140000 may be used. A current unresolved question is whether the low density clouds observed only in hydrogen are of primordial composition, or are enriched with heavy elements like the higher density absorbing systems. This can only be answered by a substantial survey with high sensitivity to faint lines, with high resolving power. Another question is the evolution with redshift in density and ionization of the intergalactic medium, as shown by the Lyman alpha clouds, which again requires a survey sensitive to weak lines over a large redshift range. The general topic of quasar absorption line spectroscopy was considered so important that it was 
selected as a key project, worthy of hundreds of hours of observing time with the first generation instruments. Even if this is carried out, it will barely scratch the surface of the topic, which STIS will be able to pursue 10-30 times faster.

Another topic selected as a key project, better suited to STIS, is the investigation of the mass distribution at the centers of active galaxies. For this, we need to know the velocity of the stellar distribution very close to the nuclei. STIS will perform long slit spectroscopy with the full angular resolution of HST in the visible, with a spectral resolving power of 20000 , providing simultaneous observations across the nuclei, with more adequate angular and spectral resolution than previously available. This will allow the determination of the mass distribution, including measuring the mass and determining the existence of black holes at the nuclei. Similar observations in the UV will measure the gas distribution and the ionization in the narrow-line region for nearby AGNs.

With its visible-blind UV area-array detectors, unique within HST, STIS will also be able to study the angular and spectral distribution of the diffuse UV background. Estimating from the Shuttle payload results by the Johns Hopkins and Berkeley groups, we see that by using a broad slit and moderate resolution grating, STIS could measure the UV continuum and spectral lines of the background. Also, in UV camera mode, the small-scale arc sec structure could be measured in individual long exposures, and the large scale structure determined by comparing observations as a function of galactic latitude. Clusters of galaxies and quasar environs could be surveyed. Much of this could be done as parallel observing, avoiding the use of prime observing time. 
Tabie Instrument Performance Sumary

Angul ar Resolution - 0.05 arcsec (Futh) - HST Limit (2 pixel sampling)

Maximum Field-of-View - 50 aresec

Aporoximate Spectral Resolutions are $10^{5}, 2 \times 10^{4}, 1200,100$

\begin{tabular}{|c|c|c|c|c|c|c|}
\hline Mode & $\begin{array}{c}\text { Wave length Range } A \\
\text { Oetector }\end{array}$ & $\begin{array}{l}\text { 1050-1700 } \\
\text { MAMA/CSI }\end{array}$ & $\begin{array}{l}1700-3000 \\
\text { MAMA/C STE }\end{array}$ & $\begin{array}{l}3000-6000 \\
C C O\end{array}$ & ${ }_{C C O}^{5500-11000}$ & \\
\hline High Resolution & $\begin{array}{l}\text { Resolving Power*( }(\lambda / \Delta \alpha) \\
\text { Effective Area } \\
\text { Angular Range }\end{array}$ & $\begin{array}{l}1,4 \times 10^{5} \\
370 \\
0.1\end{array}$ & $\begin{array}{l}1.0 \times 105 \\
560 \\
0.1\end{array}$ & $\cdots$ & -- & $\begin{array}{l}\mathrm{cm}^{2} \\
\text { arcsec }\end{array}$ \\
\hline \multirow{2}{*}{$\begin{array}{l}\text { Medium Resolution } \\
\text { (a) Wide Spectral } \\
\text { Coverage } \\
\text { (b) Wide Angular } \\
\text { Coverage }\end{array}$} & $\begin{array}{l}\text { Resolving Power } \\
\text { Effective Area } \\
\text { Angular Range }\end{array}$ & $\begin{array}{l}2.1 \times 10^{4} \\
370 \\
1.4\end{array}$ & $\begin{array}{l}2.0 \times 10^{4} \\
560 \\
1.0\end{array}$ & $\begin{array}{l}1.9 \times 10^{4} \\
3800 \\
0.6\end{array}$ & $\begin{array}{l}1.5 \times 10^{4} \\
3500 \\
0.6\end{array}$ & $\begin{array}{l}\mathrm{cm}^{2} \\
\operatorname{sicsec}\end{array}$ \\
\hline & $\begin{array}{l}\text { Resolving Power } \\
\text { Effective Area } \\
\text { instantaneous Spectral } \\
\text { Coverage of Band } \\
\text { Angular Range }\end{array}$ & $\begin{array}{l}1,4 \times 10^{4} \\
860 \\
1 / 8 \\
50\end{array}$ & $\begin{array}{l}1.6 \times 10^{4} \\
1060 \\
1 / 11 \\
50\end{array}$ & $\begin{array}{l}1.5 \times 10^{4} \\
6400 \\
1 / 13 \\
50\end{array}$ & $\begin{array}{l}1.2 \times 104 \\
6300 \\
1 / 13 \\
50\end{array}$ & $\operatorname{arcsec}$ \\
\hline Low Resolution & $\begin{array}{l}\text { Resolving Power } \\
\text { Effective Area } \\
\text { Angular Range }\end{array}$ & $\begin{array}{l}1300-2200 \\
1800 \\
50\end{array}$ & $\begin{array}{l}1000-1800 \\
1900 \\
50\end{array}$ & $\begin{array}{l}800-1600 \\
8900 \\
50\end{array}$ & $\begin{array}{l}800-1600 \\
7800 \\
50\end{array}$ & $\begin{array}{l}\mathrm{cm}^{2} \\
\text { arcsec }\end{array}$ \\
\hline $\begin{array}{l}\text { Very Low Resolution } \\
\text {-includes } 50 \times 50 \text { arc } s \\
\text { Object Ive Prism Mode }\end{array}$ & $\begin{array}{l}\text { Resolving Power } \\
\text { Effective Area } \\
\text { Angular Range }\end{array}$ & $\begin{array}{l}160-300 \\
2200 \\
50\end{array}$ & $\begin{array}{l}120-250 \\
2800 \\
50\end{array}$ & $\begin{array}{l}80-200 \\
12600 \\
50\end{array}$ & $\begin{array}{l}80-200 \\
11600 \\
50\end{array}$ & $\begin{array}{l}\mathrm{cm}^{2} \\
\text { arcssec }\end{array}$ \\
\hline \multirow{2}{*}{$\begin{array}{l}\text { Camera } \\
\text { Thincluding } \\
\text { Acquisition) }\end{array}$} & $\begin{array}{l}\text { Angular Range } \\
\text { Total I mage }\end{array}$ & $50 \times 50$ & $50 \times 50$ & $50 \times 50$ & $50 \times 50$ & $\operatorname{arcsec}$ \\
\hline & Effective Area & 3100 & 2800 & 12600 & 11600 & $\mathrm{~cm}$ \\
\hline
\end{tabular}

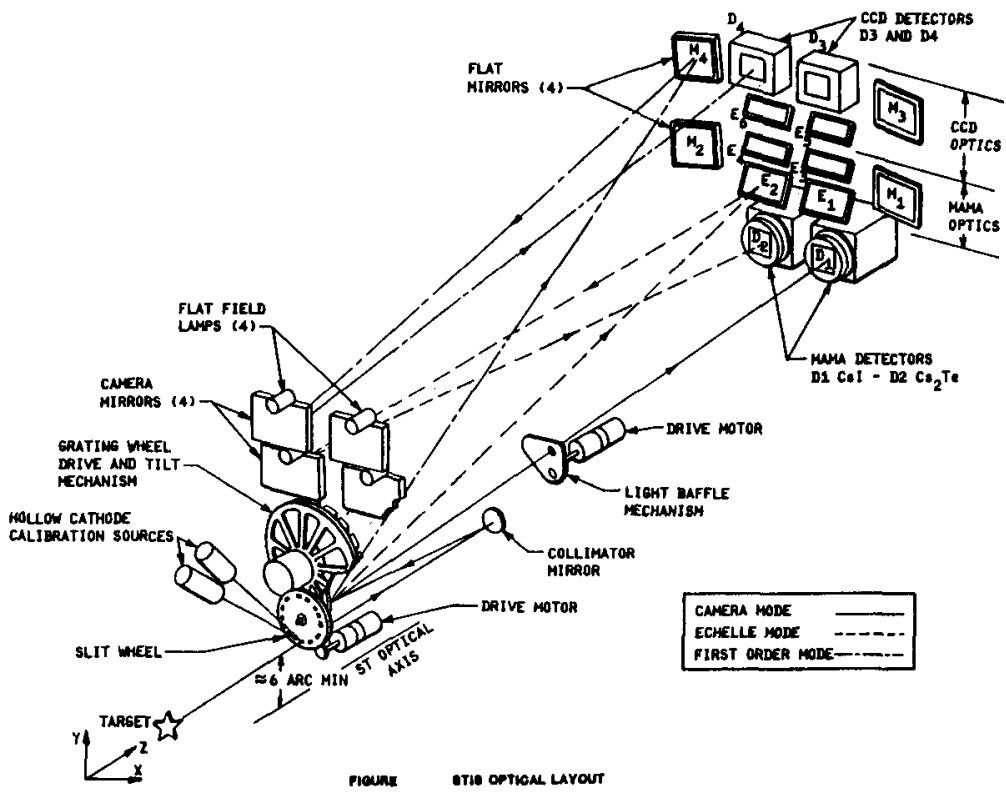

